\title{
Large Covalently Linked Fluorescent and Gold Nanoparticle Immunoprobes.
}

\author{
V. N. Joshi*, D. Mitra, M. D. Englandๆ, F. R. Furuya, R. D. Powell and J. F. Hainfeld. \\ Nanoprobes, Incorporated, 95 Horseblock Road, Unit 1, Yaphank, NY 11980. \\ I Department of Physical Sciences, Suffolk County Community College, 533 College Road, Selden, \\ NY 11784.
}

FluoroNanogold ${ }^{\mathrm{TM}}$ probes containing both a fluorescent label and the Nanogold ${ }^{\circledR}$ cluster covalently linked to a targeting molecule have been successfully used for correlative fluorescence and electron microscopy (EM) [1]. However, Nanogold is small $(1.4 \mathrm{~nm})$ and requires silver or gold enhancement for visualization [2]. This produces much greater size variability than is found in larger colloidal gold preparations. Silver enhancement may lead to non-specific background; and silver can be chemically etched by osmium tetroxide $\left(\mathrm{OsO}_{4}\right)$ fixation leading to signal loss [2]. We have prepared covalently linked combined fluorescent and larger gold nanoparticle probes for correlative microscopy that can be directly visualized by EM without the need for autometallographic enhancement. Gold nanoparticles, $2 \mathrm{~nm}$ to $40 \mathrm{~nm}$ in diameter, were stabilized and functionalized using a self-assembling coating comprising a hydrophobic chelating thiol domain to seal the gold surface from aqueous media, and hydrophilic terminal functional groups (polyethylene glycol, PEG) that biocompatibilize the gold nanoparticles and enable site-specific covalent probe conjugation. This provides high stability and a wide choice of surface properties and conjugation reactions.

$5 \mathrm{~nm}$ gold nanoparticles were prepared by direct reduction of aqueous solution of gold (III) salt with sodium borohydride in the presence of a mixture of solubilizing non-functional and protected aminefunctionalized thiols, followed by purification using sucrose density gradient (10-30\%) ultracentrifugation (Ti-41 rotor, $26,000 \mathrm{rpm}, 45 \mathrm{~min}, 5{ }^{\circ} \mathrm{C}$; the intense red band that travels $\sim 4 \mathrm{~cm}$ from the top contains $5 \mathrm{~nm}$ gold). Amine-functionalized gold particles were prepared by deprotection in methanolic hydrochloric acid. Specific reactivity was demonstrated by reaction with commercial N-hydroxy succinimido-(NHS) Cy-5.5 fluorescent dye. This conjugate was further purified by Superose-12 size exclusion chromatography (Figure 1). Unstabilized particles of this size are precipitated by $0.1 \mathrm{M}$ sodium chloride, but the coated particles remained dispersed and suspended, even in $1.0 \mathrm{M}$ sodium chloride solution. They were repeatedly centrifuged and completely resuspended without leaving any solid precipitate behind; identical treatment of conventional protein or macromolecule-stabilized colloidal gold conjugates always results in loss of a fraction as an insoluble pellet that cannot be resuspended.

Combined $5 \mathrm{~nm}$ gold-secondary antibody-Alexa Fluor 594 conjugates were prepared by covalently linking NHS- and Maleimido- activated $5 \mathrm{~nm}$ gold particles to the secondary antibody molecule, IgG and $\mathrm{F}(\mathrm{ab}$ ') respectively. NHS-modified Alexa Fluor 594 dye was then reacted with the conjugate. The product was purified using sucrose density gradient ultracentrifugation followed by gel filtration as above. The anti-rabbit-F(ab') conjugate was used as a secondary probe against a polyclonal rabbit anti-red blood cell antibody to label sheep red blood cells in suspension and the labeling was observed by fluorescence microscopy using Nikon G-2A filter set (Figure 2). The anti-mouse-IgG conjugate was used as a secondary probe with mouse anti-human AE1/AE3 primary that produced clear staining of cytokeratin in human tonsil tissue (Figure 3). The relative quantum yields for the 
fluorescent gold conjugates were $22-35 \%$ of the corresponding commercial dye labeled secondary antibodies.

\section{References}

[1] (a) F. Braet et al, Microsco. Res. Tech. 70 (2007) 230; (b) J. M. Robinson et al, J. Histochem. Cytochem. 49 (2001) 803; (c) R. D. Powell et al, Microsc. Res. Tech. 42 (1998) 2.

[2] J. F. Hainfeld and R. D. Powell, Cell Vision, 4 (1997) 408.

[3] This research was supported by NIH SBIR grant 1R43 EB008621.
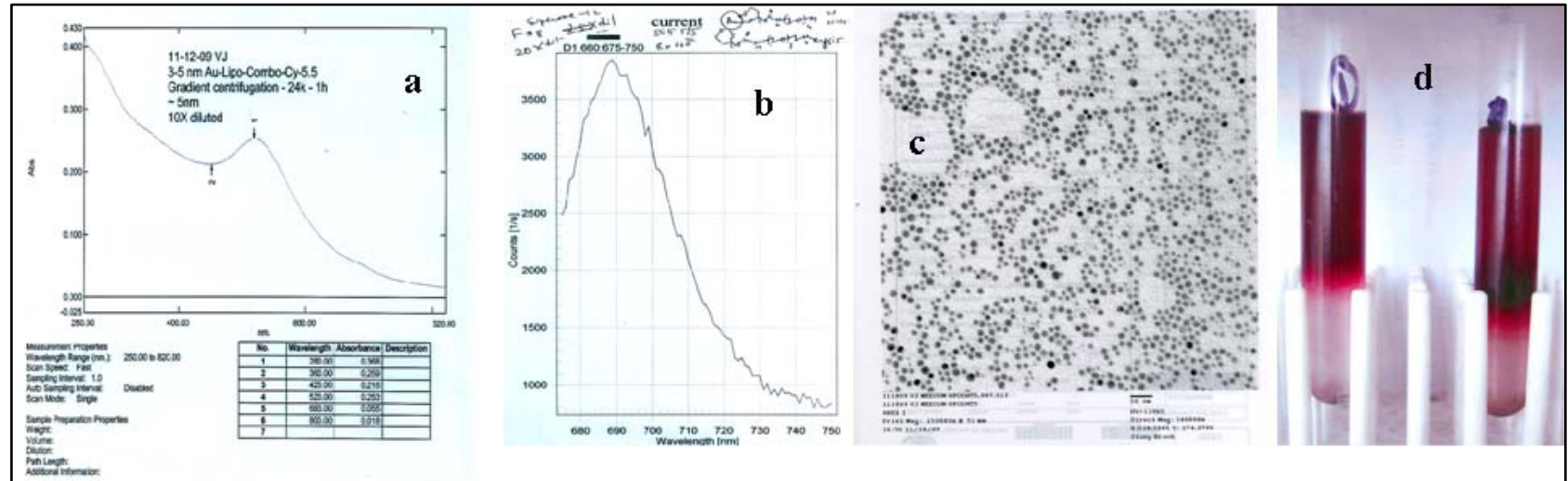

Figure 1: UV-visible, fluorescence $\left(\operatorname{Ex}_{\lambda} 660 \mathrm{~nm}\right)$ spectra, and TEM (a, b, and $\left.\mathbf{c}\right)$ of fluorescent 5 nm gold-Cy-5.5 conjugate purified by density gradient ultra-centrifugation (d) followed by size exclusion chromatography (vide supra).
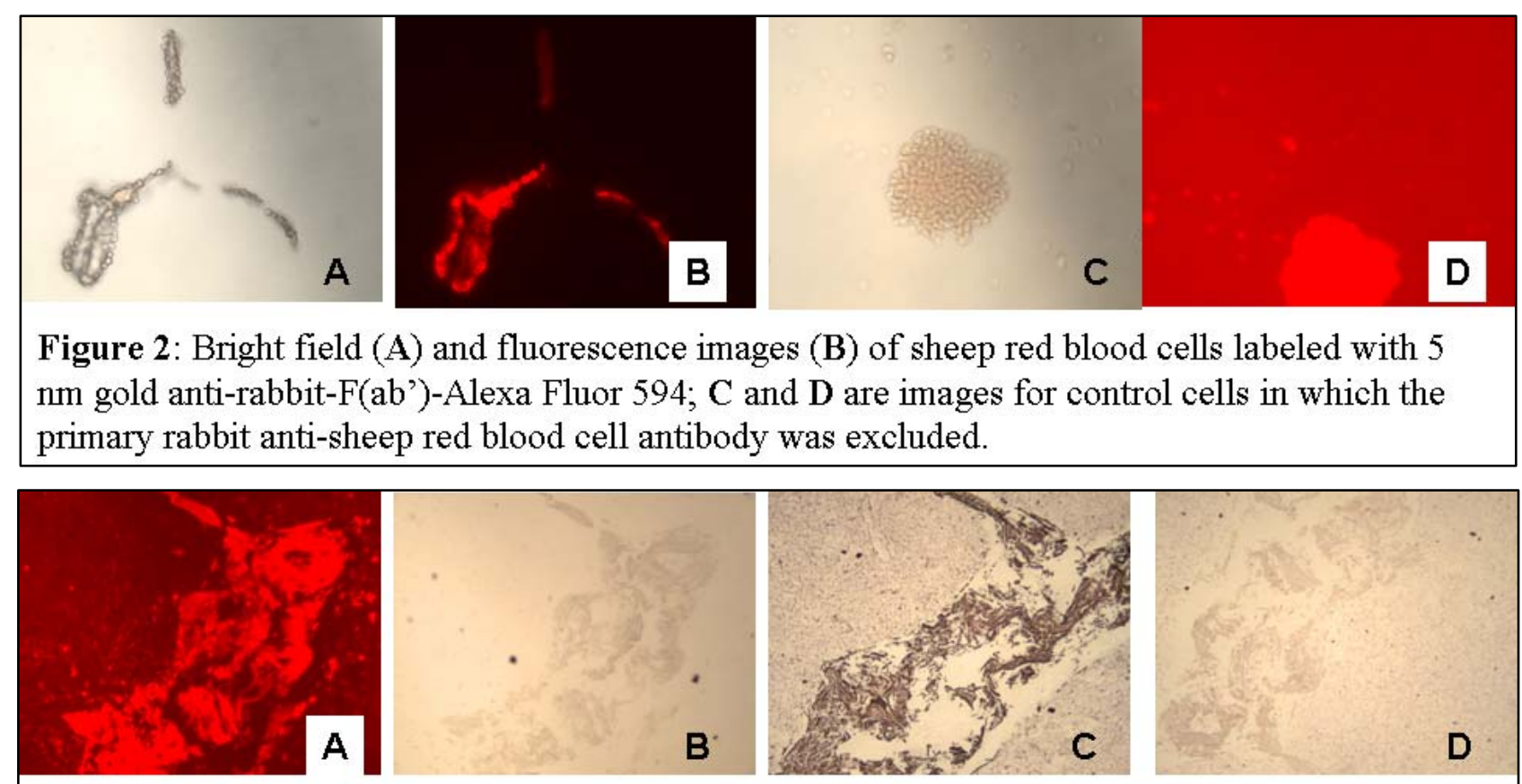

Figure 3: Fluorescence (A), bright field (B), and bright field image following silver development (C) of cytokeratin stained tonsil tissue with mouse anti-human AE1/AE3 primary and $5 \mathrm{~nm}$ gold anti-mouse-IgG-Alexa Fluor 594 secondary antibody; D is corresponding control slide in which the primary mouse anti-human antibody was excluded. 